\title{
Identification of proteins binding coding and non-coding human RNAs using protein microarrays
}

\author{
Zurab Siprashvili', Dan E Webster ${ }^{1}$, Markus Kretz ${ }^{1}$, Danielle Johnston ${ }^{1}$, John L Rinn², Howard Y Chang ${ }^{1,3}$
} and Paul A Khavari ${ }^{1,4^{*}}$

\begin{abstract}
Background: The regulation and function of mammalian RNAs has been increasingly appreciated to operate via RNA-protein interactions. With the recent discovery of thousands of novel human RNA molecules by high-throughput RNA sequencing, efficient methods to uncover RNA-protein interactions are urgently required. Existing methods to study proteins associated with a given RNA are laborious and require substantial amounts of cell-derived starting material. To overcome these limitations, we have developed a rapid and large-scale approach to characterize binding of in vitro transcribed labeled RNA to $\sim 9,400$ human recombinant proteins spotted on protein microarrays.
\end{abstract}

Results: We have optimized methodology to probe human protein microarrays with full-length RNA molecules and have identified 137 RNA-protein interactions specific for 10 coding and non-coding RNAs. Those proteins showed strong enrichment for common human RNA binding domains such as RRM, RBD, as well as K homology and CCCH type zinc finger motifs. Previously unknown RNA-protein interactions were discovered using this technique, and these interactions were biochemically verified between TP53 mRNA and Staufen 1 protein as well as between HRAS mRNA and CNBP protein. Functional characterization of the interaction between Staufen 1 protein and TP53 mRNA revealed a novel role for Staufen 1 in preserving TP53 RNA stability.

Conclusions: Our approach demonstrates a scalable methodology, allowing rapid and efficient identification of novel human RNA-protein interactions using RNA hybridization to human protein microarrays. Biochemical validation of newly identified interactions between TP53-Stau1 and HRAS-CNBP using reciprocal pull-down experiments, both in vitro and in vivo, demonstrates the utility of this approach to study uncharacterized RNA-protein interactions.

Keywords: Non-coding RNA, Microarray, p53, Ras, Staufen

\section{Background}

Functional roles for both coding and non-coding RNA molecules have been increasingly appreciated in a variety of biologic processes, including gene regulation, molecular trafficking, and protein translation [1-4]. For example, mRNAs of coding genes are increasingly recognized as targets of translational regulation by a variety of mechanisms

\footnotetext{
*Correspondence: khavari@stanford.edu

'The Program in Epithelial Biology, Stanford University School of Medicine, 269 Campus Drive, Room 2145, Stanford, CA 94305, USA

${ }^{4}$ Veterans Affairs Palo Alto Healthcare System, Palo Alto, CA 94304

Full list of author information is available at the end of the article
}

[5,6]. Additionally, thousands of long non-coding RNAs have recently been identified [7-9] and a growing number of these are being assigned discrete biologic functions [10-13]. Strategies to identify multiple RNAs binding an individual protein of interest have advanced further than those designed to identify multiple proteins binding a given RNA. Examples of the former include immunoprecipitation followed by microarray hybridization (RIP-Chip) or sequencing (RIP-Seq) $[8,14,15]$. Available approaches to accomplish the latter include RNA pull-downs in which proteins bound to biotin-labeled RNA are isolated and analyzed by techniques including mass spectrometry [16].

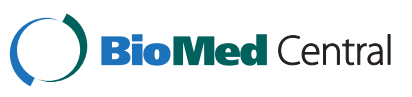


The capacity to rapidly identify multiple proteins binding to an individual RNA of interest will allow characterization of the molecular mechanisms of action and the functional role of specific RNAs in human disease.

The recent use of protein microarrays in yeast $[17,18]$ suggests a similar approach may be of utility in the more complex human setting. Commercially available human protein microarrays were designed for detection of protein-protein interactions using protein or small molecule probes. As protein microarrays permit high throughput screening for intermolecular interactions, they may also provide an alternative approach to study human protein-RNA interactions, an application in which they have not yet been reported. Here, we demonstrate a high-throughput methodology allowing rapid identification of the proteins binding to a given human RNA molecule using a protein microarray containing $\sim 9,400$ human recombinant proteins spotted in duplicate. As a result of this approach we identify previously uncharacterized interactions between HRAS RNA and CNBP protein, as well as human Stau1 and TP53 RNA and show that Stau1 influences TP53 RNA stability in the context of transcriptional blockade.

\section{Results and discussion}

To define RNA-protein interactions, we utilized sense and antisense strands for 10 RNA transcripts representing protein coding RNAs TP53, HRAS, MYC, BCL2 and non-coding RNAs PWRN1, SOX2OT, OCC1, IGF2RNC, IncRBM26 and DLEU1. The schematic diagram of the workflow used in this work is presented on Figure $1 \mathrm{~A}$ and a detailed protocol of probe preparation, labeling and hybridization conditions are included in experimental procedures section as well as in Additional file 1: Figure S1 and Additional file 2: Table S1. Briefly, the aforementioned RNAs were in vitro transcribed, labeled with Cy5 and independently probed on human protein microarrays. The labeling process was optimized in order to achieve $\sim 3$ pmol dye per $\mu \mathrm{g}$ RNA with an average efficacy of 1 dye molecule for approximately every 850 bp RNA (Additional file 2: Table S1) to minimally influence RNA native structure while yielding signal intensities that are readily visualized (Figure $1 \mathrm{~B}$ ).

In order to determine the reproducibility of RNAprotein interactions observed with this technology, we performed technical replicates of HRAS sense RNA incubation to the microarray. Comparison of the ratio of signal intensity above background of every spotted protein between the two independent replicates demonstrated suitable results with a Pearson correlation of $r^{2}=0.869$ (Figure $1 C$ ). We next analyzed the distribution of the signal within each array to establish filtering criterion for RNA-protein interaction significance. A representative distribution of signal intensities for all proteins from the TP53 sense RNA array displaying deviations from the global mean (Z-Scores) is shown on Figure 1D. It is notable that there is a large fraction of proteins that display a signal intensity ratio over background of 1, suggesting the absence of a global nonspecific fluorescence. We selected filters allowing identification of the significant RNA-protein binding events based on a Z-Score $\geq 3.0$ and a minimum signal above background of 2.5 fold. Gene lists of all significant hits from all hybridized RNAs in this study were generated (Additional file 3: Table S2), and overlaps of hits from the two technical replicates of HRAS sense RNA probed to protein array resulted in a significance of $\mathrm{p}<10^{-72}$ (Figure 1E).

Next, functional characterization of the proteins that significantly bound to RNAs used in this study was performed. Of the 9125 spotted proteins - not including spotted protein controls - only 196 proteins significantly bound to at least 1 of the 20 RNAs. These 196 RNA binding proteins were strongly enriched for protein family domains compiled in the Pfam database that have previously been identified in RNA binding, including the RNA recognition motif, RNA binding domain, zinc finger and $\mathrm{K}$ homology domains (Figure 2A). Gene Ontology (GO) terms associated with RNA binding, RNA processing, and RNA splicing among others were strongly enriched (Figure 2B). Taken together, these findings demonstrate the selectivity of this technique to detect RNAprotein interactions.

We selected a subset of these proteins that most frequently bound RNAs ( $\geq 75 \%$ of all RNAs used) for further domain analysis (Table 1). Interestingly, while 23 out of 28 of these "common RNA binding proteins" contain motifs or domains previously characterized for RNA binding capacity, several proteins such as UNG, KCNAB1, STK40, PAGE1 and NUDT16L1 contain no well-characterized RNA interaction domains despite repeated significant RNA-protein binding events observed in at least 15 out of 20 RNA incubations to the protein array (Table 1). Among these proteins, NUDT16L1 contains no defined domain at all and PAGE1 has no known function except its specific expression in variety tumors. In addition, a comparison of the 28 common RNA binding proteins to those identified by recent PAR-CL and PAR-CLIP-seq studies $[19,20]$ demonstrates that $21 / 28$ and $18 / 28$ of these proteins, respectively, demonstrate RNA binding ability including NUDT16L1.

RNA application to human protein microarrays therefore identified a set of human proteins with a broad capacity for binding to multiple RNAs that contain known RNA binding motifs as well as identified a number of new proteins for future study that contain no canonical motifs and no previously known RNA binding capacity. 


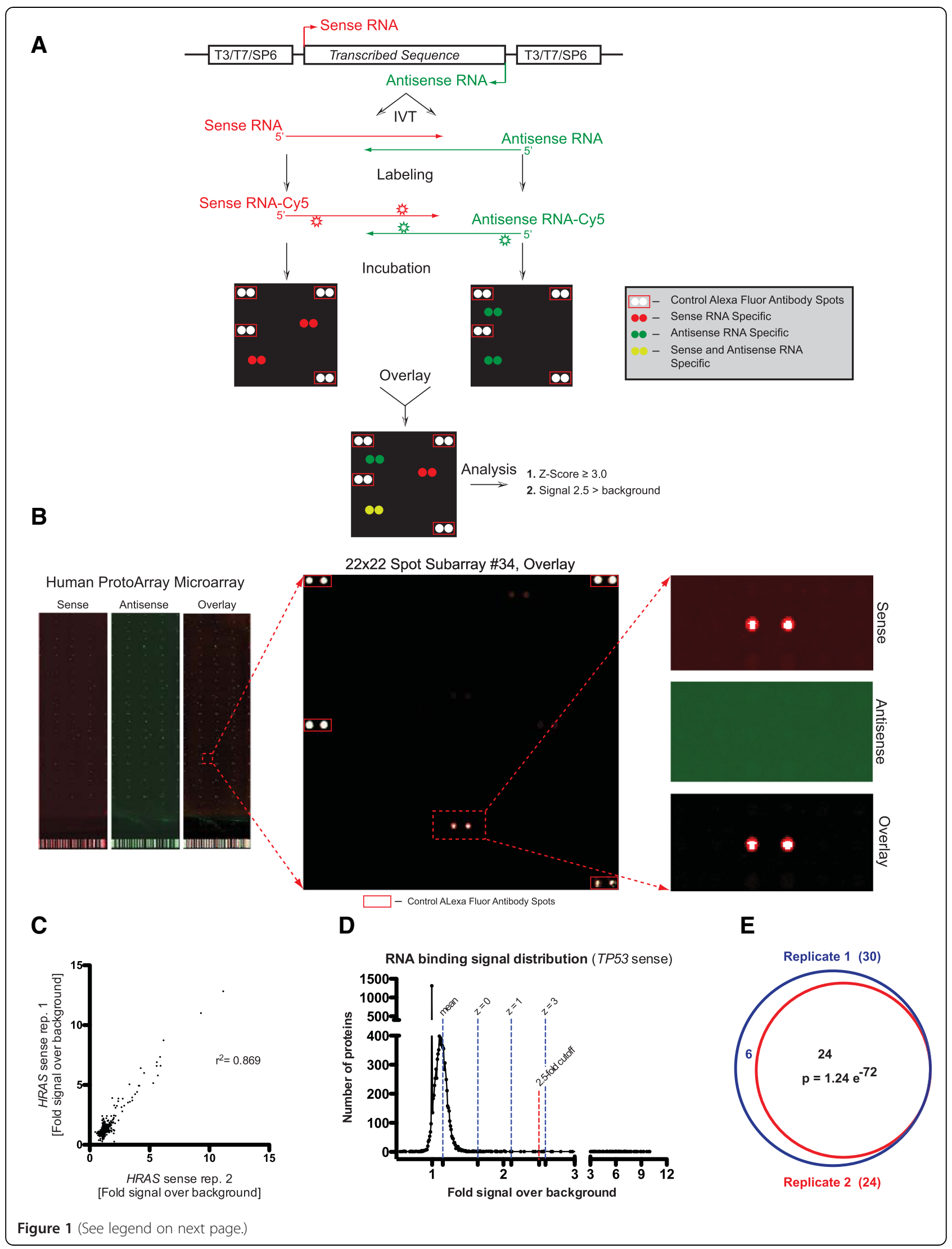




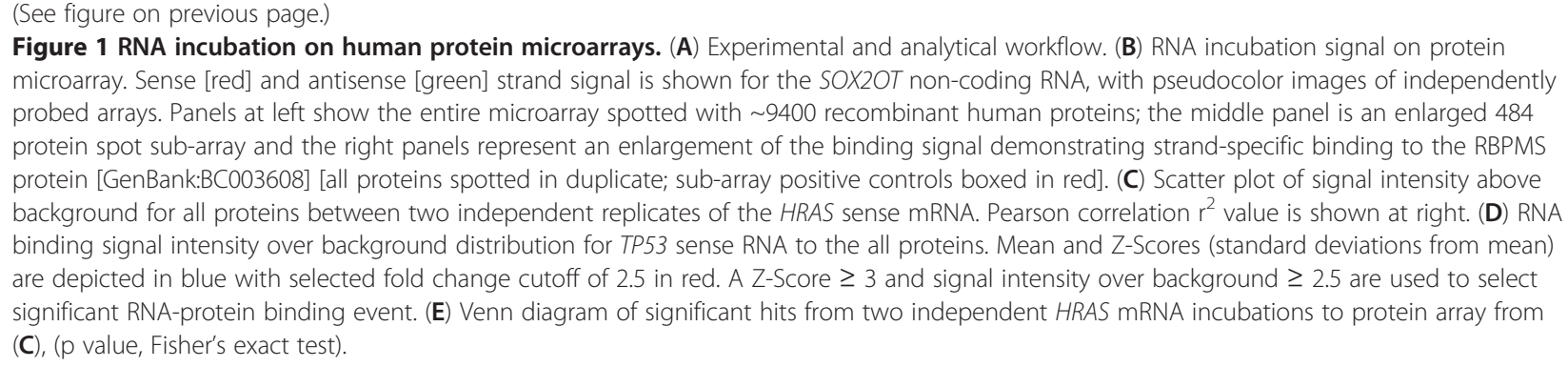

To further narrow the lists of candidate RNA-protein interactions, we selected proteins that significantly bound sense, but not antisense RNAs, although some biologically relevant interactions may take place with both sense and antisense transcripts from a given locus. This analysis resulted in 137 interactions, revealing a substantial range of protein binding between individual mRNAs and noncoding RNAs and identified specific interactions for further study (Figure 2C).

To perform biochemical validation of our findings we selected 2 of these newly identified interactions and studied RNA-protein binding using reciprocal pull-down experiments. First, Staufen1 (Stau1) was identified as a protein-binding target of TP53 mRNA using protein microarrays with signal over background 3.19-fold, Zscore 4.24 (Additional file 3: Table S2). TP53 mRNA, but not its antisense or any other RNA transcripts, displayed a specific interaction with the duplicate Stau1 protein spots (Figure $2 \mathrm{C}$ and $2 \mathrm{D}$ ). Antibody to an HA epitope-tagged Stau1 protein immunoprecipitated TP53 mRNA but not control RNAs in both in vitro and in vivo RNA-protein pull-down assays (Figure 3A and 3B). Conversely, using reciprocal RNA pull-down experiments, biotin-labeled TP53 mRNA - but not biotin-labeled controls - pulled down Stau1 protein (Figure 3C). Therefore, two-way pull-down experiments confirmed the novel TP53 mRNA-Stau1 protein interaction identified using human protein arrays.

A second interaction validation was undertaken for the RING-type $\mathrm{CCHC}$-zinc finger, nucleic acid binding protein (CNBP), which bound HRAS mRNA on the protein microarray with signal over background 3.62-fold, Z-score 3.82 (Figure 2C, Additional file 3: Table S2 and Additional file 4: Figure S2A). The biotin-labeled HRAS mRNA, but not biotin-labeled controls, pulled down CNBP protein (Additional file 4: Figure S2B). Moreover, in the complementary pull-down experiments, antibody to HA epitope-tagged CNBP protein immunoprecipitated HRAS mRNA but not control RNAs both in vitro (Additional file 4: Figure S2C), as well as in vivo (Additional file 4: Figure S2D). These two-way studies of two independent, novel targets support the validity of RNA-protein interactions detected by RNA incubation to the human protein microarray and further verifies the capability of this method.

To further address significance of TP53 association with Stau1 protein, several deletion mutants spanning the UTRs of TP53 RNA were generated and used for Stau1-HA protein pull-down (Figure $3 \mathrm{D}$ and $3 \mathrm{E}$ ). Although we could not identify any known Stau1 binding sequence motifs [21] within the TP53 UTR, pull-down experiments indicated that Stau1 protein preferentially binds within a $256 \mathrm{bp}$ sequence of the TP53 3'UTR. Moreover, repeated incubation of the TP53 open reading frame to the protein array demonstrated absence of binding signal to Stau1 duplicate spots (Additional file 4: Figure S2E), while the full-length TP53 RNA showed binding (Figure 2D), confirming the finding that Stau1 protein binding takes place within the TP53 $3^{\prime} \mathrm{UTR}$.

Stau1 protein is known to be involved in several cellular functions including RNA decay (in combination with UPF1) [22], RNA transport [23], RNA translation [24] and modulation of stress response [25] via shuffling between polysomes and stress granules. To explore consequences of Stau1 binding to TP53 RNA we studied TP53 RNA stability in STAU1 and UPF1 deficient cells. Unexpectedly, we found that although UPF1 depletion did not affect overall TP53 RNA levels (Additional file 4: Figure S2F), STAU1 knock down caused reduction in TP53 RNA half-life in actinomycin D treated primary fibroblast cells, under conditions known to halt synthesis of newly formed RNA molecules (Figure 3F). Moreover, after introduction of the full-length TP53 and TP53-ORF (lacking 3 'UTR and Stau1 protein binding site) in TP53 negative H1299 cells, STAU1 knock down caused reduction in the full-length TP53 RNA half-life but did not affect TP53-ORF RNA after actinomycin D treatment (Figure 3G). These initial findings indicate that Stau1 protein binding to TP53 RNA may play a role in preserving TP53 RNA levels in the setting of a transcriptional blockade.

The diversity of naturally occurring RNA-protein interactions is only beginning to be appreciated and RNA hybridization to human protein microarrays may be a useful complement to current platforms in a number of ways. For example, this approach can be used to 
A

Top PFAM Domains for RNA-bound Proteins

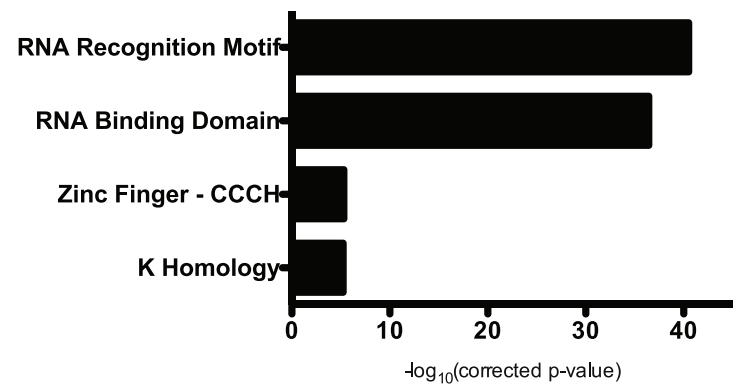

B

Top GO Terms for RNA-bound Proteins

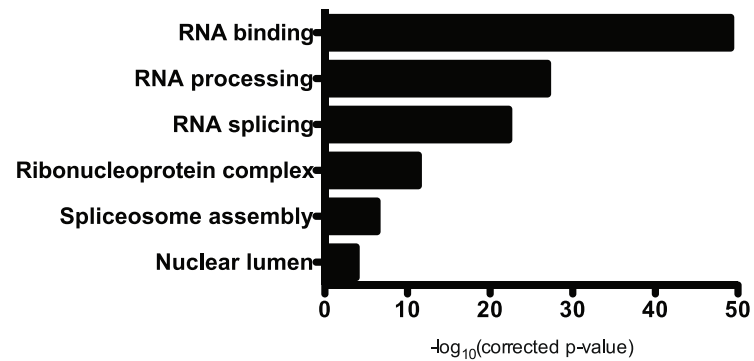

D

RNA Hybridization to Protein Arrays
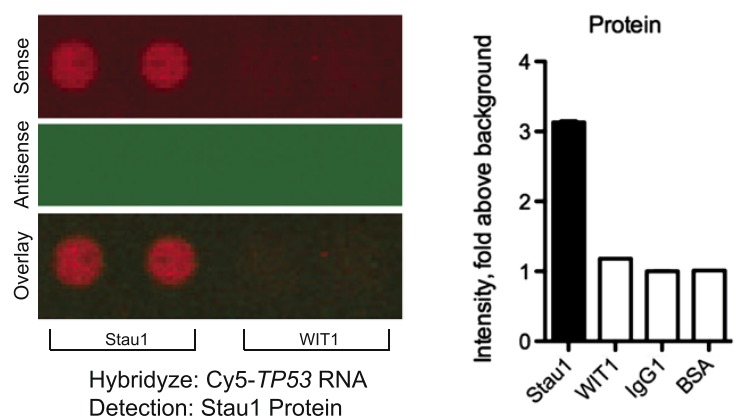

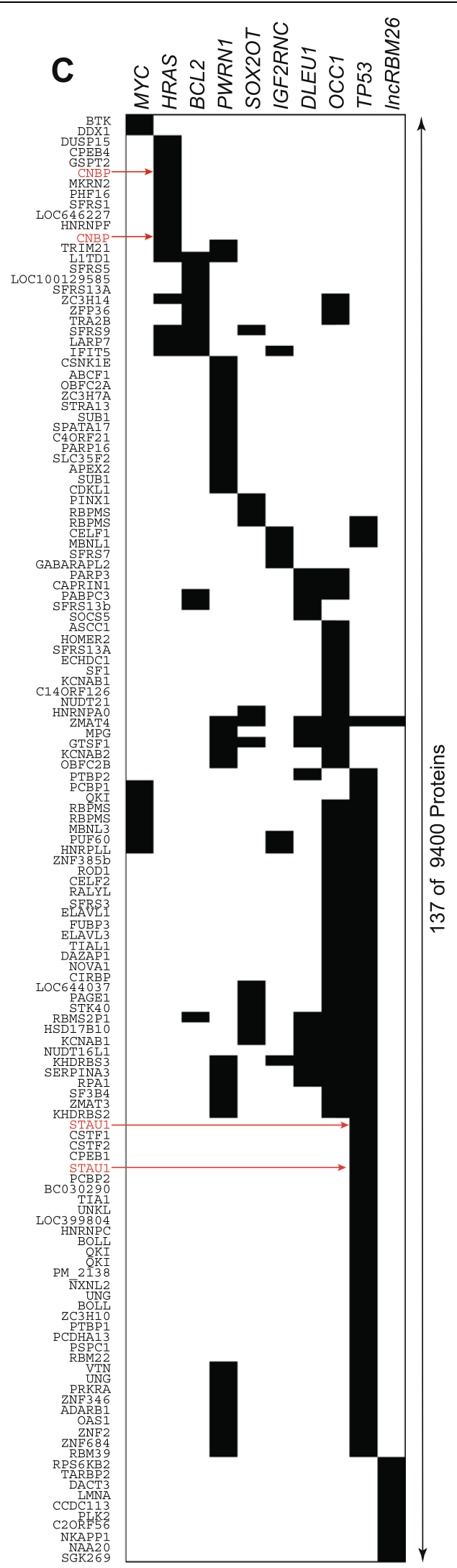

Figure 2 (See legend on next page.) 
(See figure on previous page.)

Figure 2 RNA binding proteins identified by incubation of labeled RNAs to human protein microarrays. (A) Pfam protein family domains and motifs present in proteins bound to at least 1 RNA (B) Gene ontology (GO) terms present in the same population of RNA binding proteins from (A). (C) Binary heat map representation of the RNA binding capacity to protein microarrays. Human coding and non-coding RNAs used in incubation to the microarrays are displayed in columns. The 137 out of 9400 total proteins bound by sense but not antisense strand RNA samples with $\geq 2.5$-fold above background intensity and Z-Score $\geq 3$ are displayed in rows. Stau1 and CNBP protein localization indicated with arrows. (D) Image and quantification of human protein microarray showing selective binding signal of TP53 mRNA sense strand to duplicate Stau1 protein spots. The binding signal is shown with respect to adjacent proteins spotted in the same sub-array.

Table 1 Common RNA binding proteins and their conserved domains

\begin{tabular}{|c|c|c|c|}
\hline Gene name & Protein description (NCBI Protein) & $\begin{array}{l}\text { Conserved } \\
\text { Domains }\end{array}$ & $\begin{array}{l}\text { \% of all } \\
\text { RNAs } \\
\text { significantly } \\
\text { bound }\end{array}$ \\
\hline$\overline{C I R B P}$ & Cold-inducible RNA-binding protein & RRM & $90 \%$ \\
\hline SFRS13A & FUSIP1 protein & RRM & $85 \%$ \\
\hline CPEB4 & CPEB4 protein & RRM & $85 \%$ \\
\hline PTBP2 & Polypyrimidine tract binding protein 2 & RRM & $80 \%$ \\
\hline TIAT & TIA1 protein & RRM & $80 \%$ \\
\hline TIAL1 & TIAL1 protein & RRM & $75 \%$ \\
\hline RBMS3 & RNA-binding motif, single-stranded-interacting protein 3 & RRM & $95 \%$ \\
\hline PTBP1 & Polypyrimidine tract-binding protein 1 isoform $\mathrm{c}$ & RRM & $95 \%$ \\
\hline PCBP2 & Poly $(\mathrm{rC})$-binding protein 2 isoform $b$ & PCBP_like_KH, KH-I & $100 \%$ \\
\hline NOVA1 & RNA-binding protein Nova-1 isoform 1 & PCBP_like_KH,KH-I & $90 \%$ \\
\hline PCBP2 & Poly(rC)-binding protein 2 isoform a & PCBP_like_KH, KH-I & $90 \%$ \\
\hline PCBP1 & Poly $(\mathrm{rC})$ binding protein 1 & PCBP_like_KH, KH-I & $80 \%$ \\
\hline QKI & Protein quaking isoform HQK-6 & SF1_like-KH & $95 \%$ \\
\hline QKI & Protein quaking isoform $\mathrm{HQK}-7 \mathrm{~B}$ & SF1_like-KH & $90 \%$ \\
\hline QKI & Protein quaking isoform $\mathrm{HQK}-5$ & SF1_like-KH & $80 \%$ \\
\hline KHDRBS2 & KH domain-containing,RNA-binding protein 2 & SF1_like-KH & $85 \%$ \\
\hline KHDRBS3 & KHDRBS3 protein & SF1_like-KH & $75 \%$ \\
\hline ZNF385b & ZNF385B protein & Zf-met & $90 \%$ \\
\hline MBNL1 & Muscleblind-like protein 1 isoform a & $\mathrm{Zf}-\mathrm{CCCH}$ & $75 \%$ \\
\hline ZC3H10 & Zinc finger $\mathrm{CCCH}$ domain-containing protein 10 & $\mathrm{Zf}-\mathrm{CCCH}$ & $95 \%$ \\
\hline TARBP2 & RISC-loading complex subunit TARBP2 isoform b & DSRM & $95 \%$ \\
\hline TARBP2 & RISC-loading complex subunit TARBP2 isoform a & DSRM & $100 \%$ \\
\hline RPA1 & Replication protein A 70 kDa DNA-binding subunit & RPA1N,RPA1_DBD_C & $75 \%$ \\
\hline UNG & Uracil-DNA glycosylase isoform UNG2 & UDG_F1 & $95 \%$ \\
\hline KCNAB1 & Voltage-gated potassium channel subunit beta-1 isoform 1 & Aldo_ket_red, Tas & $95 \%$ \\
\hline STK40 & Serine/threonine-protein kinase 40 & PKc_like & $75 \%$ \\
\hline PAGE1 & G antigen family B member 1 & GAGE & $75 \%$ \\
\hline NUDT16L1 & Protein syndesmos isoform 1 & None & $75 \%$ \\
\hline
\end{tabular}

RRM - RNA recognition motif, PCBP_like_KH - Poly $\mathrm{r}(\mathrm{C})$ binding protein like $\mathrm{K}$ homology domain, $\mathrm{KH}$ - K homology RNA binding domain, KH-I - K homology RNA binding domain type I, SF1_like-KH - Splicing factor K homology RNA binding domain, Zf-CCCH - Zinc-finger of CCCH [C-x8-C-x5-Cx3-H] type, DSRM - Double-stranded RNA binding motif, Aldo_ket_red - Aldo-keto reductase, Tas - predicted oxidoreductase, UDG_F1 - family 1 of Uracil-DNA glycosylase, RPA1N - replication protein A $\mathrm{N}$-terminal OB fold domain, RPA1_DBD_C - replication protein ssDNA binding domain DBD-C, PKc_like - Protein Kinase C family, GAGE - GAGE protein family. 


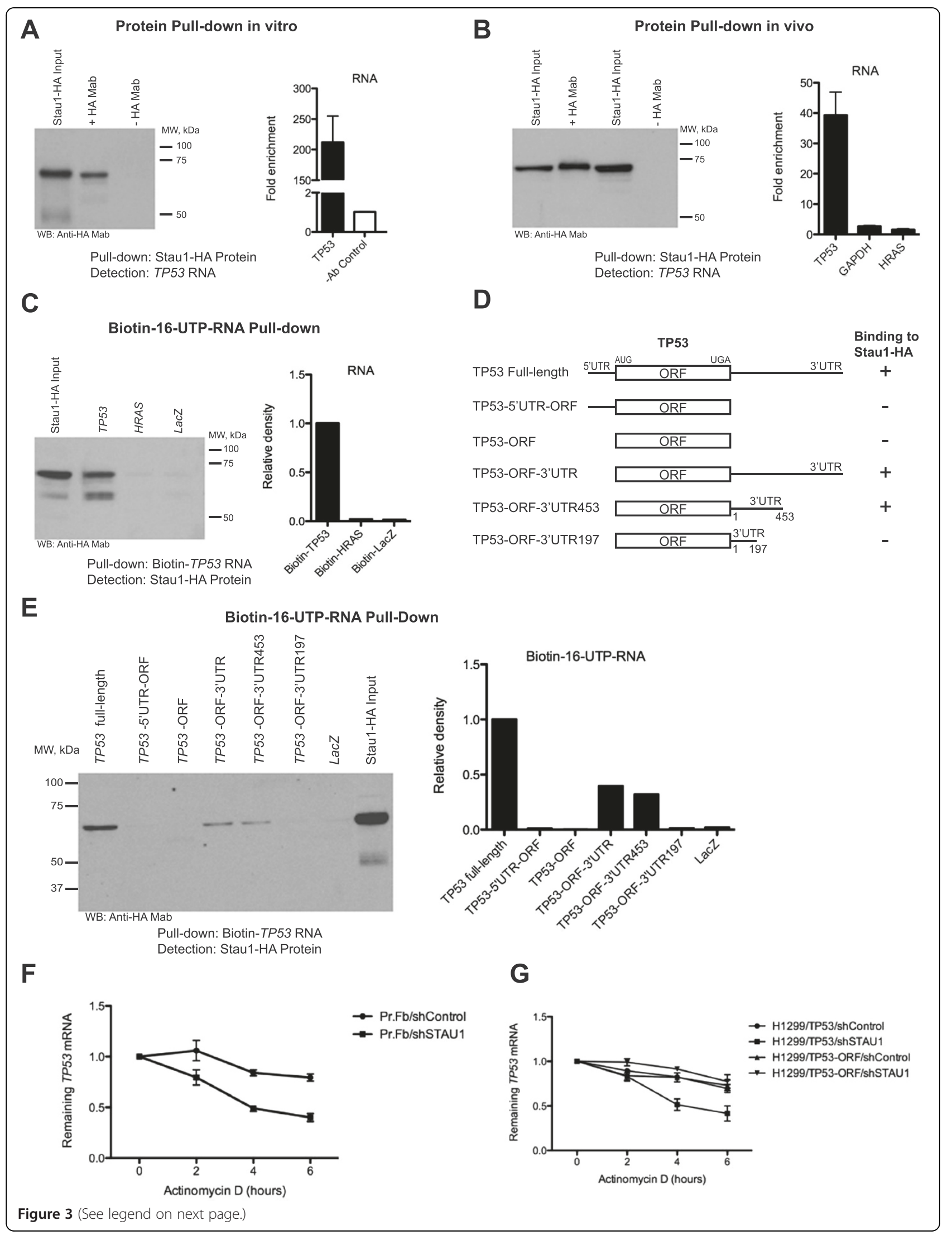


(See figure on previous page.)

Figure 3 Confirmation of RNA-protein binding on microarrays with reciprocal pull-down assays for Staufen 1 with TP53 mRNA.

(A) Stau1-HA protein pulls down TP53 mRNA in vitro after immunoprecipitation with HA Mab; immunoblots to HA-tagged Stau1 verifying Stau1 precipitation are shown on the left panel. (B) Stau1-HA protein pulls down TP53 mRNA in vivo, but not HRAS and GAPDH control RNAs after immunoprecipitation with HA Mab; immunoblots to HA-tagged Stau1 verifying Stau1 precipitation from cell extracts are shown on the left panel. (C) Pull-down of biotin labeled human TP53 mRNA in vitro, but not HRAS or LacZ mRNA precipitates associated Stau1-HA protein; densitometry quantification of the immunoblots shown on the right panel. (D) Schematic diagram of TP53 mRNA constructs. Numbering corresponds to the first nucleotide following the termination codon, defined as 1. Signs (" + " and " $-"$ ") represent ability or failure of Stau1-HA to bind TP53 RNA constructs. (E) Pull-down of biotin labeled human TP53 mRNA deletion constructs in vitro, followed by western blot analysis of the associated Stau1-HA protein; densitometry quantification of the immunoblots shown on the right panel. (F) TP53 RNA decay in Primary Fibroblasts after actinomycin D treatment. (G) Comparison of full-length TP53 and TP53-ORF (lacking Staufen 1 interaction domain) RNA decay in TP53 negative H1299 cells after actinomycin D treatment.

gain mechanistic insight into newly identified long noncoding RNAs by understanding the proteins to which they bind. The ability to test the binding capability of any coding or non-coding RNA to thousands of proteins simultaneously will significantly improve the pace of mechanistic analyses of RNAs. In this regard, it may provide a complement to biotin-labeled RNA pulldown/mass spectrometry-based approaches, which have proven successful in identifying RNA binding proteins [8] but require substantial amounts of cell-derived starting material and are laborious and time-consuming. RNA hybridization to human protein microarrays, in contrast, requires minimal amounts of RNA and can be completed in less than one day. However, the use of entirely recombinant components for this method precludes the detection of RNA-protein interactions that 1) require intact protein complexes, 2) require posttranslational modifications of protein, and 3) take place with proteins not spotted on the array.

RNA hybridization to human protein microarrays may also help to understand proteins that bind and regulate the stability, localization, or translational control of protein coding transcripts. In this regard, Stau1, a known RNA-binding protein implicated in both RNA stability and localization [22-24], was shown in this work to regulate TP53 in an as yet previously uncharacterized way. Similarly, the binding of CNBP to HRAS RNA may also modulate Ras function. Moreover, it is intriguing to note that human genetic disorders arising from mutations in both CNBP (Myotonic Dystrophy [26]) and HRAS (Costello Syndrome [27]), while displaying distinctive features, both display profound abnormalities in muscle tissue. Similarly, we observed the Prader-Willi Syndrome associated long non-coding RNA, PWRN1, to bind the SPATA17 protein, which has been linked to apoptosis of spermatogenic cells [28]. As hypogonadism is a known symptom of Prader Willi Syndrome, this observation may provide a functional link between this non-coding RNA and its associated human disease. Incubation of specific human RNAs of interest to human protein microarrays may therefore help characterize mechanisms of RNA function and may also stimulate efforts to identify potential uncharacterized links in the pathogenesis of human disease.

\section{Conclusions}

Here we describe a refined methodology for rapid and large-scale identification of novel human RNA-protein interactions. RNA hybridization to human protein microarrays described here offers several attractive features. First, the reagents required are readily available, consisting of minimal amounts of in vitro transcribed RNA and standardized, commercially available protein microarrays. Second, RNA hybridization to human protein microarrays does not require large-scale cell culture for protein isolation and mass spectrometry, and hence it is far less laborious than current RNA chromatography techniques. Third, this technique is rapid, taking less than a day to complete. Biochemical verification of newly identified RNA-protein interactions using this technique via reciprocal and independent pull-down experiments performed here moreover suggests that there are many undiscovered human-RNA protein interactions and that this approach may be helpful in identifying them.

\section{Methods}

\section{Plasmid vectors and expression constructs}

Plasmid vectors containing full-length transcribed sequences for coding and non-coding RNAs were obtained from Open Biosystems (Thermo Scientific) and are described in detail in Additional file 2: Table S1. All plasmids except pDNRLIB contain T7, T3 or SP6 promoters for sequencing and in vitro RNA production. pDNRLIB-DLEU1 plasmid contains the T7 promoter for sense RNA transcription. To produce antisense DLEU1 RNA, a 945 bp DLEU1 sequence was recloned from pDNRLIB into PSPORT1 vector using EcoRI and XhoI sites and pSPORT1-DLEU1 was used for antisense RNA production via SP6 promoter. For in vivo RNA production we constructed a small eukaryotic expression vector pSPARTA containing the human PGK promoter controlling expression of the transcribed sequence and puromycin resistance gene for selection in both prokaryotes and eukaryotes, which is under control of the synthetic bacterial EM7 and viral CMV promoter 
(Additional file 1: Figure S1). The TP53 mRNA sequence [GenBank:NM_000546] was recloned from the original pSPORT1-TP53 vector as a SalI-ClaI fragment of $2560 \mathrm{bp}$ into Sall-EcoRV of pSPARTA. The TP53 deletion mutants containing $5^{\prime} \mathrm{UTR}$ and $197 \mathrm{bp}$ of $3^{\prime} \mathrm{UTR}$ were constructed by flanking TP53 fragments with either HindIII-XhoI or EcoRI-XhoI and cloning into pcDNA3 expression vector to generate pcDNA3-TP53-ORF-5'UTR and pcDNA3-TP53ORF-3'UTR197. The constructs containing TP53-ORF, TP53-ORF-3'UTR (full-length) and TP53-ORF-3'UTR453 fragments were generated by direct amplification and TA cloning into pcDNA3.1TOPO expression vector. Numbering for $3^{\prime} U T R$ deletion constructs corresponds to the first nucleotide following the termination codon, defined as 1. The full-length TP53 expression construct used for transfection of H1299 cells was constructed by recloning HindIII-NotI fragment from pSTARTA-TP53 into pcDNA3 in order to take advantage of the neomycin selection cassette in H1299 cells.

Human V-Ha-Ras homolog [GenBank:BC006499] was recloned from pOTB7 vector as 1146 bp EcoRI-XhoI fragment into EcoRV-XhoI of pSPARTA. Both plasmids pSPARTA-TP53 and pSPARTA-HRAS were used in pulldown experiments in vivo as described below. In vivo expression constructs for Staul and CNBP were generated using pcDNA3.1Hygro plasmid (Invitrogen). The ORFs of Stau1 and CNBP were flanked with $3 \mathrm{x}$ hemagglutinin (HA) tag at $3^{\prime}$ end for Staul and $5^{\prime}$ end for CNBP using PCR techniques and the following primers:

STAUF, 5'TTTTAAGCTTACCATGTCTCAAGTTCAAG TGCAAGTT.

STAUHAR,

5'TTTTCTCGAGTCAGGCGTAATCGGGCACGTCGTA GGGATAGCTTCCTGCATAATCAGGGACGTCATAGG GATAGCCAGCATAGTCAGGCACATCGTATGGGTAG CACCTCCCACACACAGACA.

CNBPHAF, 5'TTTTAAGCTTACCATGTACCCATACGA

TGTGCCTGACTATGCTGGCTATCCCTATGACGTCC CTGATTATGCAGGAAGCTATCCCTACGACGTGCCC GATTACGCCAGCAGCAATGAGTGCTTC AAG. CNBPR, 5'TTTTCTCGAGTTAGGCTGTAGCCTCAAT TGTG.

The epitope tagged fragments were cloned in HindIIIXhoI sites of pcDNA3.1Hygro and the final constructs pcDNA3.1Hygro-STAU1-HA and pcDNA3.1Hygro-HACNBP were used for RNA pull-down studies.

\section{In vitro RNA production and labeling}

For the 10 RNA expression sequences used in this study, RNAs for coding (TP53, MYC, HRAS, BCL2) and noncoding (OCC1, IGF2RNC, PWRN1, DLEU1, IncRBM26, $S O X 2 O T)$ genes were in vitro transcribed in both sense and antisense directions using T7, T3 or SP6 promoters (Additional file 2: Table S1). First, plasmid DNA was digested with enzymes immediately flanking the transcribed sequence and $4 \mu \mathrm{g}$ of linear DNA was used for in vitro transcription in $50 \mu \mathrm{l}$ total volume consisting of: 1x Transcription buffer (Promega), $10 \mathrm{mM}$ DTT (Promega), $1 \mathrm{mM}$ NTP (Invitrogen), 40 units RNAseOUT (Invitrogen) and 60 units RNA polymerases (T7, T3 or SP6). The reaction was carried out at $37^{\circ} \mathrm{C}$ for 4 hours after which DNA was digested by addition of 2 units DNAse I at $37^{\circ} \mathrm{C}$ for 15 minutes. Next, RNA was phenol-chloroform extracted and after ethanol precipitation measured using NanoDrop 1000 spectrophotometer (Thermo Scientific) and visualized using denaturing agarose gel-electrophoreses (Additional file 1: Figure S1C).

RNA labeling for microarray incubations was performed using Label IT $\mu$ Array Cy5 labeling kit (Mirus). We first carefully optimized the labeling procedure in order to achieve between 1 to $3 \mathrm{Cy} 5$ dyes covalently attached to RNAs used. This was accomplished via the following modifications from the original manufacturers protocol: the total reaction volume was kept at $25 \mu \mathrm{l}$, the ratio of RNA:Label IT Cy5 reagent at 10:1 (w:v) and reaction time not more then 30 minutes at $37^{\circ} \mathrm{C}$. Briefly, $5 \mu \mathrm{g}$ RNA in water was mixed with $5 \mu \mathrm{L}$ Label IT Cy5, diluted 1 to 10 in water to obtain a final volume of $25 \mu \mathrm{l}$ and incubated 30 minutes at $37^{\circ} \mathrm{C}$. The reaction was stopped by addition $2.5 \mu \mathrm{l}$ of $10 \mathrm{x}$ STOP buffer (Mirus). The volume was increased to $100 \mu \mathrm{l}$ with water, supplemented with glycogen (Invitrogen) to final concentration of $0.2 \mu \mathrm{g} / \mu \mathrm{l}$, mixed and RNA ethanol precipitated in the presence of $0.5 \mathrm{M} \mathrm{NaCl}$ at $-20^{\circ} \mathrm{C}$ for at least 1 hour. The labeled RNA was extensively washed in $70 \%$ ethanol, dried and resuspended in $16 \mu \mathrm{l}$ water. RNA labeling density was evaluated using NanoDrop 1000 spectrophotometer (Thermo Scientific) and visualized using denaturing agarose gel-electrophoreses.

The efficacy of Cy5 dye incorporation was calculated as dye density (pmol Dye: $\mu \mathrm{g}$ RNA) and RNA Base:Dye ratio. To calculate the dye density following formula was used:

pmol Dye : $\mu \mathrm{g}$ RNA $=\mathrm{A}_{\text {dye }} / \mathrm{e}_{\text {dye }}(\mathrm{pmol}) / \mathrm{M}_{\mathrm{RNA}},(\mu \mathrm{g})$, where

$A_{\text {dye }}-$ Cy5 absorbance at $I_{\max }$ (excitation wavelength) $649 \mathrm{~nm}$,

$\mathrm{e}_{\text {dye }}-$ Cy5 dye extinction coefficient $250000 \mathrm{M}^{-1} \mathrm{~cm}^{-1}$, $\mathrm{M}_{\text {RNA }}$ - RNA amount ( $\left.\mu \mathrm{g}\right)$.

Base:Dye ration was calculated using following formula: $\left(\mathrm{A}_{\text {base }} \mathrm{X} \mathrm{e}_{\text {dye }}\right) /\left(\mathrm{A}_{\text {dye }} \mathrm{X} \mathrm{e}_{\text {base }}\right)$, where

$A_{\text {base }}=A_{260}-\left(A_{\text {dye }} \times\right.$ C.F.260 $)-$ RNA base absorbance,

$\mathrm{A}_{260}$ - absorbance of the nucleic acid, C.F.260 -

correction factor for $\mathrm{Cy} 5=0.05$,

$\mathrm{e}_{\text {base }}$ - RNA extinction coefficient $8250 \mathrm{M}^{-1} \mathrm{~cm}^{-1}$ 
The RNA labeling density and Base/Dye labeling ratio for each 20 sense and antisense RNAs used in this work is presented in Additional file 2: Table S1, with an RNA labeling efficacy required of 1 Cy5 dye per $700-1200$ bp RNA.

RNA labeling with Biotin-16-UTP was performed during in vitro transcription. First, plasmid DNA containing transcribed sequence was digested with enzymes immediately flanking the insert and $4 \mu \mathrm{g}$ of linear DNA was used for RNA biotinilation reaction in $50 \mu$ total volume consist of: 1x Transcription buffer (Promega), $1 \mathrm{mM}$ ATP, 1mM CTP, 1mM GTP, 0.95 mM UTP, $0.05 \mathrm{mM}$ Biotin-16-UTP (Roche), 10 mM DTT (Promega), 40 units RNAseOUT (Invitrogen) and 60 units RNA polymerases (T7, T3 or SP6). The reaction was carried out at $37^{\circ} \mathrm{C}$ for 4 hours after which DNA was digested by addition of 2 units DNAse I at $37^{\circ} \mathrm{C}$ for 15 minutes. Next, RNA was phenol chloroform extracted, and after ethanol precipitation, characterized using NanoDrop 1000 spectrophotometer (Thermo Scientific) and visualized using denaturing agarose gel-electrophoreses (Additional file 1: Figure S1D).

\section{ProtoArray processing and analysis}

For RNA incubation, ProtoArray Human Protein Microarray v5.0 (Invitrogen, cat\# PAH052520) was used. Prior to incubation, each microarray was equilibrated first to $4^{\circ} \mathrm{C}$ overnight and then to $25^{\circ} \mathrm{C}$ for at least for 15 minutes. The microarray slide was assembled in a Gentel SIMplex 16 Multi-Array System device (Gentel biosciences, cat\# 41007 ) with custom modifications including a modified bottom gasket and top spacers (Additional file 1: Figure S1A). The bottom gasket was cut out from silicone slab as a rectangular seal with the following dimensions in $\mathrm{mm}$ : outer $65 \mathrm{~L} \times 25 \mathrm{~W} \times 4 \mathrm{H}$, inner $59 \mathrm{~L} \times 20 \mathrm{~W} \times 4 \mathrm{H}$. Top spacers were cut out from a polycarbonate piece as a rectangular shape with the following dimensions in $\mathrm{mm}$ : Upper spacer $5 \mathrm{~L} \times 22 \mathrm{~W} \times 1 \mathrm{H}$, lower spacer $12 \mathrm{~L} \times 22 \mathrm{~W} \times 1 \mathrm{H}$. First, the microarray slide was placed inside a Gentel SIMplex 16 Multi-Array device bottom holder piece, next the silicone gasket was carefully placed on the top of the slide and the device top piece held in place with tightening screws. The slide surface was blocked in $0.7 \mathrm{~mL}$ blocking buffer BL: $40 \mathrm{mM}$ Tris- $\mathrm{HCl}$ (pH 8.0), 1\% BSA (w/v) (globulin free, Sigma, cat\# A7638), $100 \mu \mathrm{g} / \mathrm{ml}$ Yeast tRNA, $20 \mu \mathrm{g} / \mathrm{mL}$ heparin and $1 \mathrm{mM}$ DTT for 1 hour at room $\mathrm{T}^{\circ} \mathrm{C}$ with gentle agitation. After completion of the blocking step, $10 \mathrm{pmol}$ Cy5 labeled RNA was added to $0.7 \mathrm{~mL}$ binding buffer BB: $40 \mathrm{mM}$ Tris- $\mathrm{HCl}$ ( $\mathrm{pH}$ 8.0), $150 \mathrm{mM}$ sodium chloride, $0.5 \mathrm{mM}$ magnesium acetate, $10 \mu \mathrm{g} / \mathrm{ml}$ Yeast tRNA, $10 \mu \mathrm{g} /$ $\mathrm{mL}$ heparin, $1 \mathrm{mM}$ DTT, 0.01\% Igepal CA-630, 5\% glycerol, 0.2 units/ $\mu$ RNAseOUT. The blocking buffer was replaced with binding buffer containing labeled RNA and microarray slides incubated in the dark at $25^{\circ} \mathrm{C}$ for 1 hour with gentle agitation. Following RNA incubation binding buffer was removed and washing steps were implemented using 3 times exchange of $0.75 \mathrm{~mL}$ WB buffer: $40 \mathrm{mM}$ Tris$\mathrm{HCl}(\mathrm{pH}$ 8.0), $150 \mathrm{mM}$ sodium chloride, $0.5 \mathrm{mM}$ magnesium acetate, $10 \mu \mathrm{g} / \mathrm{ml}$ Yeast tRNA, $10 \mu \mathrm{g} / \mathrm{mL}$ heparin, $1 \mathrm{mM}$ DTT, $0.01 \%$ Igipal 40, 5\% Glycerol, 0.2 units/ $\mu \mathrm{l}$ RNAseOUT for at least 5 minutes each. Finally, the microarray slide was washed in $0.75 \mathrm{~mL}$ washing buffer WBF: $40 \mathrm{mM}$ Tris- $\mathrm{HCl}$ ( $\mathrm{pH} 8.0$ ), $150 \mathrm{mM}$ sodium chloride, $0.5 \mathrm{mM}$ Magnesium acetate, $10 \mu \mathrm{g} / \mathrm{ml}$ Yeast tRNA, $10 \mu \mathrm{g} / \mathrm{mL}$ heparin, $1 \mathrm{mM}$ DTT, at least 3 times for $5 \mathrm{~min}$ utes each. After the last wash, the WBF buffer was removed partially in order to prevent the slide from drying prematurely and slide holder device disassembled immediately. The probed microarray slide was placed in ArrayIt microarray high-speed centrifuge and the residual WBF buffer removed via a 30 seconds of centrifugation. The dry slide was scanned at $635 \mathrm{~nm}$ (Cy5) using a GenePix 4000B Microarray scanner (Molecular Devices) immediately after or at least within 2 hours of the completion of the incubation. All raw and processed data is publicly available at the Gene Expression Omnibus under accession GSE34794 (http://www.ncbi.nlm.nih.gov/geo/query/acc.cgi?token=ttehdwscqcgckrg\&acc=GSE34794). For the visualization process, the array images from antisense RNA incubations were pseudocolored green and overlaid with the sense RNA incubation. The intensity of the $635 \mathrm{~nm}$ wavelength signal at each spotted protein location was determined with GenePix Pro 6.1 software (Molecular Devices). To quantify RNA-protein interactions, the intensity of $635 \mathrm{~nm}$ signal (F635) was divided by the local background intensity (B635) at each of the duplicate spots for a given protein. Data was filtered based on signal above the background for each of the duplicate feature to be greater than 2.5 fold and Z-Score $\geq 3$ from the global mean signal from all of the spotted proteins. In order to select sense-specific protein interactions, the signal generated from antisense RNA incubations were calculated based on 2.5 fold above the background and Z-Score $\geq 3$. Significant hits from the antisense were subtracted from the significant hits in the sense RNA. Hierarchical clustering analysis was performed by Cluster 3.0 (Eisen Lab) and visualized with TreeView. Gene Ontology analysis and PFAM domain analysis of RNA binding proteins was performed with DAVID [29] using as a background a universe of gene list of all proteins spotted on the microarray and Benjamini-Hochberg correction of the $p$ value. The $\mathrm{p}$ value of the Venn diagram illustrating overlap of two independent microarray incubations was calculated using Fisher's exact test.

\section{RNA-protein complex pull-downs}

RNA-protein complex pull-downs were performed in two complementary directions: first, by immunoprecipitation 
of target protein with following associated RNA detection using qPCR technique. Second, by pulling-down biotin labeled RNA and detecting RNA associated protein via western blot analyses.

\section{In vitro protein IP with subsequent RNA detection}

For in vitro protein RNA complex immunoprecipitation both Stau1-HA and HA-CNBP proteins were in vitro translated using rabbit reticulocyte lysate system (Promega) in accordance with manufacturer's recommendations. The full-length human TP53 and HRAS mRNA transcripts were in vitro transcribed as described in above. Prior to RNA-protein complex formation, $20 \mu \mathrm{l}$ Protein G Dynabeads (Invitrogen) were saturated in $500 \mu \mathrm{l}$ buffer IPB containing $40 \mathrm{mM}$ Tris- $\mathrm{HCl}$ (pH 8.0), $150 \mathrm{mM}$ sodium chloride, $0.5 \mathrm{mM}$ Magnesium acetate, $20 \mu \mathrm{g} / \mathrm{mL}$ heparin, $1 \mathrm{mM}$ DTT, 0.01\% Igepal CA-630, $5 \%$ Glycerol, supplemented with $0.5 \%$ BSA, $100 \mu \mathrm{g} / \mathrm{ml}$ Yeast tRNA and protease inhibitors complete mini (Roch) and mixed with $2.5 \mu \mathrm{g}$ anti-HA antibody (HA.11, clone 16B12, Covance) for 1 hour with continuous agitation at $25^{\circ} \mathrm{C} .12 .5 \mu \mathrm{l}$ of in vitro translated protein was mixed with 250 ng RNA in $500 \mu \mathrm{l}$ buffer IPB supplemented with 0.2 units/ $\mu \mathrm{l}$ RNAseOUT and incubated 1 hour at $25^{\circ} \mathrm{C}$ with gentle agitation. After one wash of HA-bound protein G Dynabeads in IPB buffer proteinRNA complex was added to the beads and incubated for 1 hour at room $\mathrm{T}^{\circ} \mathrm{C}$. As a control, pre-blocked protein G Dynabeads lacking HA antibody was added to the same amount of protein-RNA complex and processed identically to the sample tube. After completion of the protein capture on Dynabeads, five separate washes for ten minutes duration each were implemented using IPB buffer. Magnetic beads were resuspended in $100 \mu \mathrm{l} \mathrm{IPB}$ and $10 \%$ removed for protein analysis via western. The RNA from the residual mixture containing protein-RNA complex was extracted with phenolchloroform then ethanol precipitated for resuspension in $11.5 \mu \mathrm{l}$ water in order to use for cDNA synthesis with iScript cDNA Synthesis kit (Bio-Rad). After completion of the reverse transcription, DNA samples were subjected to qPCR using Maxima SYBR Green qPCR master mix (2x, Fermentas) and gene specific primers sets:

TP53_F: 5'CCAGCCAAAGAAGAAACCAC TP53_R: 5'TGAGTTCCAAGGCCTCATTC HRAS_F: 5'AGCAGATCAAACGGGTGAAG HRAS_R: 5'AGCCAGGTCACACTTGTTCC

The qPCR was performed on Stratagene Mx3000P QPCR system (Agilent Technologies) and results were analyzed with MxPro QPCR software v. 4.1.

\section{In vivo protein IP with subsequent RNA detection}

For in vivo protein-RNA complex pull-down experiments 293T cells were transfected with expression vectors pcDNA3.1Hygro-STAU1-HA, pSPARTA-TP53, and pcDNA3.1Hygro-HA-CNBP pSPARTA-HRAS in $10 \mathrm{~cm}$ plates using FuGENE 6 transfection reagent (Roche) in accordance with the manufacturer's instructions. Cells were collected 48 hours post-transfection in 2 volumes (v/v cell pallet) of Buffer A: $10 \mathrm{mM}$ Tris- $\mathrm{HCl}$ ( $\mathrm{pH} 7.5)$, $0.1 \mathrm{mM}$ EDTA, $1 \mathrm{mM}$ DTT, $1 \mathrm{mM}$ PMSF and protease inhibitors complete mini (Roch) and incubated 15 minutes on $4^{\circ} \mathrm{C}$. While cells were incubated on ice Protein G Dynabeads were blocked and bound to $\mathrm{HA}$ mab as described above using IPB buffer. After 15 minutes Igepal CA-630 was added to cells to a final concentration of $0.01 \%$ and incubated additional 5 minutes at $4^{\circ} \mathrm{C}$. Next, cells were subjected to two freeze-thaw cycles by incubating on isopropanol/dry ice bath for 30 seconds and immediately thawing at $37^{\circ} \mathrm{C}$. After completion of the lyses, $1.5 \mu \mathrm{l}$ of RNAseOUT (Invitrogen) was added for every $100 \mu \mathrm{l}$ lysate and subjected to centrifugation at $1,000 \mathrm{~g}$ for 5 minutes at $4^{\circ} \mathrm{C}$. The cytosolic fraction was removed and kept at $4^{\circ} \mathrm{C}$, while to remaining pellet Buffer $\mathrm{B}$ was added equal to the volume of original Buffer A supplemented with 2.5 units of DNAse I for every $100 \mu \mathrm{l}$ of original cell pellet. Buffer B is composed of: $50 \mathrm{mM}$ Tris- $\mathrm{HCl}(\mathrm{pH}$ 7.5), $300 \mathrm{mM}$ sodium chloride, $1 \mathrm{mM}$ Magnesium acetate, $1 \mathrm{mM}$ DTT, 10\% Glycerol (v/v), 1 mM PMSF and protease inhibitors complete mini (Roch). The lysate was passed through 27 gauge needle, spun at 10,000g for 15 minutes at $4^{\circ} \mathrm{C}$ and, after adding Igepal CA-630 to a final concentration of $0.01 \%$, combined with cytosolic fraction. Next, the combined lysate was split in two parts and treated with $20 \mu \mathrm{l}$ Protein G Dynabeads with or without HA Mab for 1 hour at room $\mathrm{T}^{\circ} \mathrm{C}$. The magnetic beads were subjected to at least 5 washing cycles using $500 \mu \mathrm{l}$ IPB buffer and, after collection in $100 \mu \mathrm{l}$ buffer, $1 / 10$ of the volume was saved for protein analysis via western and from the rest of the pull-down RNA extracted using TRIzol reagent (Invitrogen) in accordance with the manufacturer's instructions. The RNA pallet was resuspended in $11.5 \mu \mathrm{l}$ in order to use for cDNA synthesis with iScript cDNA Synthesis kit (Bio-Rad). After completion of the reverse transcription, DNA samples were subjected to qPCR using Maxima SYBR Green qPCR master mix (2x, Fermentas) and gene specific primers sets indicated above together with the control primers:

\section{GAPDH_F: 5' GAAGAGAGAGACCCTCACTGCTG GAPDH_R: 5'ACTGTGAGGAGGGGAGATTCAGT}

The qPCR was performed on Stratagene Mx3000P QPCR system (Agilent Technologies) and results were analyzed with MxPro QPCR software v. 4.1. 
In vitro RNA pull-down with subsequent protein detection For in vitro RNA pull-down TP53 full-length or deletion mutants TP53-5'UTR-ORF, TP53-ORF, TP53-ORF3'UTR, TP53-ORF-3'UTR453, TP53-ORF-3'UTR197, HRAS and LacZ mRNAs were labeled with biotin-16UTP as described above and Additional file 1: Figure S1D. The control LacZ RNA was prepared as $1200 \mathrm{bp}$ fragment of the full-length $L a c Z$ via in vitro transcription of the EcoRV digested pcDNA3.1HygroLacZ (Invitrogen) with T7 polymerase in order to match the average length of the sample RNAs used in this work. The $5 \mu \mathrm{l}$ in vitro translated Stau1-HA or HA-CNBP was incubated with $1 \mu \mathrm{g}$ biotin-16-UTP labeled TP53, HRAS, or LacZ in IPB buffer for 30 minutes at $25^{\circ} \mathrm{C}$. During this reaction, $5 \mu \mathrm{l}$ of MyOne Streptavidin T1 Dynabeads (Invitrogen) were exchanged to IPB buffer using magnetic stand and added to protein RNA complex. The mixture was incubated an additional 30 minutes and subjected to five wash cycles of 5 minutes each using $500 \mu \mathrm{l}$ IPB buffer. After the last wash, magnetic beads were resuspended in $12 \mu \mathrm{l}$ protein loading buffer, RNA bound protein separated by SDS-PAGE and detected with anti-HA Mab by western blot analysis.

\section{RNA interference}

siRNA oligonucleotide duplexes used in this work were synthesized by Dharmacon (Thermo Scientific). $1 \times 10^{6}$ Pimary human Fibroblasts were electroporated with 1 nmol siRNA nucleotides using Amaxa Human Dermal Fibroblast nucleofection kit (Lonza) following manufacturer's instruction. The siRNA oligonucleotides were used in this work:

\section{siControl: 5' GUAGAUUCAUAUUGUAAGGUU siUPF1 A: $5^{\prime}$ CAGCGGAUCGUGUGAAGAA siUPF1 C: $5^{\prime}$ GCAGCCACAUUGUAAAUCA}

The vectors for pGIPZ shRNA targeting Staul were designed and purchases through Open Biosystems (Thermo Scientific) catalog number RHS4531-NM_017452. The efficiency of knockdown was evaluated using following oligonucleotides:

\section{STAU_F: $5^{\prime}$ ATGGTATCGGCAAGGATGTG STAU_R:5' AGACATTGGTCCGTTTCCTG UPF1_F: $5^{\prime}$ ATATGCCTGCGGTACAAAGG UPF1_R: 5' AGCTCAATGGCGATCTCATC}

\section{Tissue culture}

All of the experimental procedures were carried out in accordance with the local ethics commission. Primary human dermal fibroblasts were isolated from neonatal dermis and cultured at early passage in DMEM supplemented with 10\% FBS. H1299 non-small cell lung carcinoma cell line deficient for TP53 was cultured in RMPI1640 supplemented with 10\% FBS. H1299 cells were transfected with pcDNA3-TP53 full-length or pcDNA3.1TOPO-TP53-ORF constructs and selected for 3 to 5 days in $500 \mu \mathrm{g} / \mathrm{mL}$ neomycin. Next, cells were transduced with pGIPZ-STAU1 virus and 36 hours later selected with $1 \mu \mathrm{g} / \mathrm{mL}$ puromycin for 48 hours. For RNA stability analysis, cells were plated 24 hours before at $40-50 \%$ confluence in 6 well plate and next day treated with $5 \mu \mathrm{g} / \mathrm{mL}$ actinomycin D (Sigma) for the indicated times. Total RNA was prepared and gene expression analyses as described above.

\section{Additional files}

Additional file 1: Figure S1. Protein microarray incubation device and RNAs used for this work. (A) Modified Gentel SIMplex 16 device with microarray slide and assembly components. Schematic diagram of the custom-made silicone gasket and spacer with main dimensions indicated. (B) Expression vector PSPARTA. hPGK - human phosphoglycerate kinase promoter, SV40 polyA - simian virus 40 polyadenylation signal, bGlob polyA beta-globin polyadenylation signal, Puromycin - resistance gene, EM7 bacterial promoter, CMV Enh/Prom - cytomegalovirus enhancer promoter, Ori - origin of replication. Unique site depicted in black. Polylinker sites are in red. (C) Denaturing agarose gel electrophoresis of sense and antisense RNAs used in this work. M - RiboRuler RNA ladder (bp): 6000, 4000, 3000, 2000, 1500, 1000, 500, 200. (D) Denaturing agarose gel electrophoresis of biotin-16-UTP labeled RNAs TP53, HRAS, LacZ (1.2kb fragment of LacZ, experimental procedures). M RiboRuler RNA ladder (bp): 6000, 4000, 3000, 2000, 1500, 1000, 500, 200.

Additional file 2: Table S1. Expression constructs and promoters used for the strand-specific RNA sequence production. The efficiency of RNA labeling with Cy5 dye was calculated as described in Experimental Procedures.

Additional file 3: Table S2. Significant RNA binding proteins for all sense and antisense RNAs used in this work. Z-Score $\geq 3$ and signal intensity above background $\geq 2.5$ was used to filter RNA-protein binding events as described in the text.

Additional file 4: Figure S2. Confirmation of RNA-protein binding on microarrays. The reciprocal pull-down assays for CNBP with HRAS mRNA. (A) Quantitation images of human microarray showing selective binding signal of HRAS mRNA sense strand to duplicate CNBP protein spots. The incubation signal shown with respect to adjacent protein controls in the same sub-array. (B) Pull-down of biotin labeled HRAS mRNA in vitro, but not TP53 or LacZ precipitates associated HA-CNBP protein; densitometry quantification of the immunoblots shown (right). (C) HA-CNBP protein pulls down HRAS mRNA in vitro after immunoprecipitation with HA Mab; immunoblots to HA-tagged CNBP verifying CNBP precipitation are shown on the left panel. (D) HA-CNBP protein pulls down HRAS mRNA in vivo, but not control TP53 and GAPDH mRNAs after immunoprecipitation with HA Mab; immunoblots to HA-tagged CNBP verifying CNBP precipitation from cell extracts are shown (left). (E) Incubation of TP53-ORF mRNA sense strand lacking $5 /$ and $3 /$ UTR regions to human protein microarrays. Panel at left shows the entire microarray spotted with 9400 recombinant human proteins; the middle panels represent an enlargement of the sub-array containing Stau1 and WIT1 proteins. Note absence of the Stau1-TP53 mRNA association signal in comparison to Figure 2D when full-length TP53 mRNA was probed. [all proteins spotted in duplicate; Stau1 and WIT1 spots boxed in white; sub-array positive controls boxed in red]. The quantification of the incubation results shown on the right. (F) Contrary to STAU1 depletion, UPF1 KD has no affect on TP53 RNA decay in Primary Fibroblasts.

\section{Abbreviations}

RNA: Ribonucleic acid; RIP-Chip: RNA-binding protein immunoprecipitationmicroarray profiling; RIP-Seq: RNA-binding protein immunoprecipitation- 
sequencing; RRM: RNA recognition motif; RNP: Ribonucleic acid-protein; UTR: Untranslated region; PGK: Phosphoglycerate kinase; EM7: Prokaryotic promoter; CMV: Cytomegalovirus; ORF: Open reading frame; PCR: Polymerase chain reaction; UTP: Uridine-5'-Triphosphate; ATP: Adenosine-5'-Triphosphate; CTP: Cytidine-5'-Triphosphate; GTP: Guanosine $-5^{\prime}$-Triphosphate; DTT: Dithiothreitol; Tris: 2-Amino-2-hydroxymethyl-propane-1,3-diol; tRNA: Transfer RNA; EDTA: Ethylenediaminetetraacetic acid; PMSF: Phenylmethanesulfonylfluoride; SDS-PAGE: Sodium dodecyl sulfate polyacrylamide gel electrophoresis; PFAM: Database of protein families; GO: Gene ontology; DAVID: The Database for annotation, visualization and integrated discovery.

\section{Competing interests}

The authors declare no competing financial interests.

\section{Authors' contribution}

ZS and DJ carried out experiments including RNA labeling, protein array probing and functional studies, DW participated in data analysis and statistical calculations, MK participated in the design of the study and RNA interference experiments. PK directed the research, and with JR and $\mathrm{HC}$, conceived and participated in its design, coordination and helped to write the manuscript. All authors read and approved the final manuscript.

\section{Acknowledgements}

We thank C. Lee, R. Flockhart, A. Zehnder, and G. Kim for helpful discussions and presubmission review. This work was supported by the U.S. Veterans Affairs Office of Research and Development and by National Institutes for Health and National Institute of Arthritis and Musculoskeletal and Skin Diseases grant [AR49737 to PAK].

\section{Author details}

${ }^{1}$ The Program in Epithelial Biology, Stanford University School of Medicine, 269 Campus Drive, Room 2145, Stanford, CA 94305, USA. ${ }^{2}$ Department of Stem Cell and Regenerative Biology, Harvard University, Cambridge, MA 02138, USA. ${ }^{3}$ Howard Hughes Medical Institute, Stanford, CA 94305, USA. ${ }^{4}$ Veterans Affairs Palo Alto Healthcare System, Palo Alto, CA 94304.

Received: 18 May 2012 Accepted: 11 November 2012 Published: 16 November 2012

\section{References}

1. Wilusz JE, Sunwoo H, Spector DL: Long noncoding RNAs: functional surprises from the RNA world. Genes Dev 2009, 23(13):1494-1504.

2. Mattick JS: RNA regulation: a new genetics? Nat Rev Genet 2004, 5(4):316-323.

3. Ponting $\mathrm{CP}$, Oliver $\mathrm{PL}$, Reik W: Evolution and functions of long noncoding RNAs. Cell 2009, 136(4):629-641.

4. Bartel DP: MicroRNAs: target recognition and regulatory functions. Cell 2009, 136(2):215-233.

5. Pesole G, Mignone F, Gissi C, Grillo G, Licciulli F, Liuni S: Structural and functional features of eukaryotic mRNA untranslated regions. Gene 2001, 276(1-2):73-81.

6. Mignone F, Gissi C, Liuni S, Pesole G: Untranslated regions of mRNAs. Genome Biol 2002, 3(3):REVIEWS0004.

7. Guttman M, Amit I, Garber M, French C, Lin MF, Feldser D, Huarte M, Zuk O, Carey BW, Cassady JP, et al: Chromatin signature reveals over a thousand highly conserved large non-coding RNAs in mammals. Nature 2009, 458(7235):223-227.

8. Khalil AM, Guttman M, Huarte M, Garber M, Raj A, Rivea Morales D, Thomas K, Presser $A$, Bernstein $B E$, van Oudenaarden $A$, et al: Many human large intergenic noncoding RNAs associate with chromatin-modifying complexes and affect gene expression. Proc Natl Acad Sci USA 2009, 106(28):11667-11672.

9. Pauli A, Rinn JL, Schier AF: Non-coding RNAs as regulators of embryogenesis. Nat Rev Genet 2011, 12(2):136-149.

10. Rinn JL, Kertesz M, Wang JK, Squazzo SL, Xu X, Brugmann SA, Goodnough LH, Helms JA, Farnham PJ, Segal E, et al: Functional demarcation of active and silent chromatin domains in human HOX loci by noncoding RNAs. Cell 2007, 129(7):1311-1323.

11. Rastan S: X chromosome inactivation and the Xist gene. Curr Opin Genet Dev 1994, 4(2):292-297.
12. Yotova IY, Vlatkovic IM, Pauler FM, Warczok KE, Ambros PF, Oshimura M, Theussl HC, Gessler M, Wagner EF, Barlow DP: Identification of the human homolog of the imprinted mouse Air non-coding RNA. Genomics 2008, 92(6):464-473.

13. Martianov I, Ramadass A, Serra Barros A, Chow N, Akoulitchev A: Repression of the human dihydrofolate reductase gene by a non-coding interfering transcript. Nature 2007, 445(7128):666-670.

14. Townley-Tilson WH, Pendergrass SA, Marzluff WF, Whitfield ML: Genome-wide analysis of mRNAs bound to the histone stem-loop binding protein. RNA 2006, 12(10):1853-1867.

15. Hafner M, Landthaler M, Burger L, Khorshid M, Hausser J, Berninger P, Rothballer A, Ascano M Jr, Jungkamp AC, Munschauer M, et al: Transcriptome-wide identification of RNA-binding protein and microRNA target sites by PAR-CLIP. Cell 2010, 141(1):129-141.

16. Slobodin B, Gerst JE: A novel mRNA affinity purification technique for the identification of interacting proteins and transcripts in ribonucleoprotein complexes. RNA 2010, 16(11):2277-2290.

17. Tsvetanova NG, Klass DM, Salzman J, Brown PO: Proteome-wide search reveals unexpected RNA-binding proteins in Saccharomyces cerevisiae. PLoS One 2010, 5(9):e12671

18. Scherrer T, Mittal N, Janga SC, Gerber AP: A screen for RNA-binding proteins in yeast indicates dual functions for many enzymes. PLoS One 2010, 5(11):e15499.

19. Baltz AG, Munschauer M, Schwanhausser B, Vasile A, Murakawa Y, Schueler M, Youngs N, Penfold-Brown D, Drew K, Milek M, et al: The mRNA-bound proteome and its global occupancy profile on protein-coding transcripts. Mol Cell 2012, 46(5):674-690.

20. Castello A, Fischer B, Eichelbaum K, Horos R, Beckmann BM, Strein C, Davey $\mathrm{NE}$, Humphreys DT, Preiss T, Steinmetz LM, et al: Insights into RNA biology from an atlas of mammalian mRNA-binding proteins. Cell 2012, 149(6):1393-1406

21. Kim YK, Furic L, Parisien M, Major F, DesGroseillers L, Maquat LE: Staufen1 regulates diverse classes of mammalian transcripts. EMBO J 2007, 26(11):2670-2681.

22. Kim YK, Furic L, Desgroseillers L, Maquat LE: Mammalian Staufen1 recruits Upf1 to specific mRNA 3/UTRs so as to elicit mRNA decay. Cell 2005, 120(2):195-208.

23. Kiebler MA, Hemraj I, Verkade P, Kohrmann M, Fortes P, Marion RM, Ortin J, Dotti CG: The mammalian staufen protein localizes to the somatodendritic domain of cultured hippocampal neurons: implications for its involvement in mRNA transport. I Neurosci: the official journal of the Society for Neuroscience 1999, 19(1):288-297.

24. Dugre-Brisson S, Elvira G, Boulay K, Chatel-Chaix L, Mouland AJ, DesGroseillers L: Interaction of Staufen 1 with the 5/ end of mRNA facilitates translation of these RNAs. Nucleic Acids Res 2005, 33(15):4797-4812.

25. Thomas MG, Martinez Tosar L, Loschi M, Pasquini JM, Correale J, Kindler S, Boccaccio GL: Staufen recruitment into stress granules does not affect early mRNA transport in oligodendrocytes. Mol Biol Cell 2005, 16(1):405-420.

26. Chen W, Wang Y, Abe Y, Cheney L, Udd B, Li YP: Haploinsuffciency for Znf9 in Znf9+/- mice is associated with multiorgan abnormalities resembling myotonic dystrophy. J Mol Biol 2007, 368(1):8-17.

27. Aoki Y, Niihori T, Kawame H, Kurosawa K, Ohashi H, Tanaka Y, Filocamo M, Kato K, Suzuki Y, Kure S, et al: Germline mutations in HRAS protooncogene cause Costello syndrome. Nat Genet 2005, 37(10):1038-1040.

28. Deng Y, Hu LS, Lu GX: Expression and identification of a novel apoptosis gene Spata17 (MSRG-11) in mouse spermatogenic cells. Acta Biochim Biophys Sin (Shanghai) 2006, 38(1):37-45.

29. da Huang W, Sherman BT, Lempicki RA: Systematic and integrative analysis of large gene lists using DAVID bioinformatics resources. Nat Protoc 2009, 4(1):44-57.

doi:10.1186/1471-2164-13-633

Cite this article as: Siprashvili et al:: Identification of proteins binding coding and non-coding human RNAs using protein microarrays. BMC Genomics 2012 13:633. 